Dementia

and Geriatric

Cognitive Disorders
Dement Geriatr Cogn Disord 2020;49:2-7

DOI: $10.1159 / 000506332$
Received: December 12, 2019

Accepted: February 1, 2020

Published online: March 30, 2020

\title{
Diagnostic Impact of CSF Biomarkers in a Local Hospital Memory Clinic Revisited
}

\author{
Leo Boelaarts $^{a}$ Jos F.M. de Jonghe ${ }^{a} \quad$ Philip Scheltens $^{b}$ \\ ${ }^{a}$ Department of Geriatric Medicine, NoordWest Hospital Group, Alkmaar, The Netherlands; ${ }^{\mathrm{b}}$ Alzheimer Center, \\ Amsterdam University Medical Centers, Amsterdam, The Netherlands
}

\section{Keywords}

Alzheimer's disease - Cerebrospinal fluid biomarkers . Geriatric medicine - Outpatient memory clinic

\begin{abstract}
Background/Aims: Research guidelines on predicting and diagnosing Alzheimer's disease (AD) acknowledge cerebrospinal fluid (CSF) levels as pivotal biomarkers. We studied the usefulness of CSF biomarkers in the diagnostic workup of patients in a geriatric outpatient memory clinic of a communitybased hospital, attempted to determine a cutoff age for the use of CSF biomarkers in this group of patients, and compared the total $\tau / A \beta$ ratio as an alternative CSF diagnostic rule with the usual rules for interpreting CSF levels. Methods: This was a prospective study of consecutively referred patients. Inclusion criteria were described on the basis of previous study results in the same setting. The CSF tool was applied either to differentiate between $A D$ and no AD or to increase certainty having made the diagnosis of $A D$. Clinicians were asked to judge whether the CSF results were helpful to them or not. Results: The reasons to use the CSF tool in the diagnostic workup were in 78/106 patients to decide between the diagnosis "AD" and "no $A D$ " and in 28/106 patients to increase the certainty regarding the diagnosis. In $75 \%$ of cases the CSF levels were considered diagnostically helpful to the clinicians. Results in the present setting suggest 65 years as the cutoff age to use CSF as a diagnostic tool. The sensitivity and specificity of the total $\tau / A \beta$ ratio using the clinical diag-
\end{abstract}

\begin{tabular}{ll}
\hline KARGER & (c) 2020 The Author(s) Karger \\
& Published by S. Karger AG, Basel Open access \\
karger@karger.com & This article is licensed under the Creative Commons Attribution- \\
NonCommercial-NoDerivatives 4.0 International License (CC BY- \\
Nww.karger.com/dem \\
Nsage and distribution for commercial purposes as well as any dis- \\
tribution of modified material requires written permission.
\end{tabular}

nosis as the gold standard were at least as good as the usual categorization rule. Conclusions: Our study results corroborate earlier findings that the CSF tool is of added value to the diagnostic workup in daily clinical practice outside tertiary referral centers. CSF levels can best be used in patients under 66 years of age. Given the limited use of this tool in settings outside research facilities, we recommend that the usefulness of CSF biomarkers is studied in a multicenter study. When in the future CSF levels can be reliably measured in plasma, this may become even more relevant.

(c) 2020 The Author(s)

Published by S. Karger AG, Basel

\section{Introduction}

Cerebrospinal fluid (CSF) biomarkers, amyloid- $\beta_{1-42}$ $(\mathrm{A} \beta)$, total $\tau$ and $\tau$ phosphorylated at threonine-181 $(\mathrm{p}-\tau)$ have been extensively studied to determine their value in diagnosing Alzheimer's disease (AD) and to predict conversion from subjective cognitive disorder and mild cognitive impairment (MCI) to AD. These studies have resulted in updated research guidelines positioning CSF parameters as pivotal biomarkers in predicting and diagnosing AD [1-4]. In most outpatient memory clinics outside research facilities, CSF biomarkers are not part of the daily practice in diagnosing cognitive disorder although it has been suggested that CSF biomarkers might here also be of clinical value [5-8]. 
Between 2005 and 2008 we studied the impact of CSF biomarkers in the diagnostic process in a general hospital outpatient memory clinic of a geriatric medicine department and found that the added value of CSF biomarkers seemed largest for patients who presented diagnostic doubts between $\mathrm{AD}$ and no dementia. Data in this sample with a mean age of 72.5 years strongly suggested diagnostic usefulness was modest in older patients probably due to mixed pathology [5].

Based on these results we continued to use CSF biomarkers but preferably in younger patients, i.e. under 71 years, and provided no extensive white matter hyperintensities, other extensive vascular pathology, or other clinically relevant neuropathology was present on MRI.

In the present study we evaluated the success of this policy by asking the following questions: (1) Do CSF biomarkers in patients under 71, without imaging evidence of major vascular or other neuropathology, have clinical usefulness in our diagnostic program in addition to the regular workup? (2) Do CSF levels in patients under 71 years show a more typical profile in cognitively healthy and $\mathrm{AD}$ patients, and can we find an indication of a cutoff age for the use of the CSF tool? And (3), does the total $\tau$ / $\mathrm{A} \beta$ ratio have added value when interpreting CSF levels in individual cases [9]?

\section{Patients and Methods}

This was a prospective study including all consecutive patients referred to an outpatient memory clinic of a community-based hospital between 2010 and 2016. The regular diagnostic workup consisted of taking a medical history, physical and neurological examination, laboratory tests, ECG, brain MRI, and an extensive test battery covering all domains of cognitive function. All diagnostic information was discussed in a multidisciplinary team resulting in a clinical consensus diagnosis [10]. Patients were diagnosed with probable or possible AD if they fulfilled NINCDSADRDA criteria [11], frontotemporal lobe dementia using the criteria of Neary et al. [12], vascular dementia $(\mathrm{VaD})$ by the NINCDS-AIREN criteria [13], dementia with Lewy bodies using the criteria of McKeith et al. [14], or dementia with unknown etiology. Nondemented patients were either categorized as "no cognitive disorder" (NCD), symptoms of a cognitive disorder caused by a developmental disorder, a psychiatric or neurological disorder, or patients were diagnosed with amnestic or multidomain $\mathrm{MCI}$, according to the Petersen criteria $[15,16]$. In case of diagnostic doubt additional diagnostic procedures were considered, e.g. EEG, FDG PET, dopamine transporter scan or CSF biomarkers.

A lumbar puncture (LP) with CSF analysis was requested either to increase diagnostic certainty regarding the diagnosis of $\mathrm{AD}$ or to differentiate between $\mathrm{AD}$ and "no AD." CSF was preferably not collected in patients over 70 years. Patients had to give written consent for use of the CSF material for additional research ends. Exclusion criteria for measurement of CSF biomarkers were exten- sive white matter hyperintensities as defined by a Fazekas score of 3 [17], other vascular pathology sufficient to diagnose $\mathrm{VaD}$, or another clinically relevant neuropathology.

The CSF biomarker tool is an Alzheimer diagnostic instrument giving either a result "AD" or "no AD." "No AD" means that CSF levels of $A \beta$, total $\tau$ and $p-\tau$ are within normal limits, hence no $A D$ pathology. We used in this study 2 preCSF diagnosis groups to analyze the data: $\mathrm{AD}$ and no- $\mathrm{AD}$ patients. The no- $\mathrm{AD}$ group was comprised of NCD, psychiatric disorder, and developmental disorder patients.

The patients were split up in 3 age groups, a group that could be characterized as "young," <66 years, a group of patients that were outside the preferred age range, i.e. over 70 years, and a group in between.

The local ethics committee approved the study.

\section{CSF Analysis}

CSF was obtained by LP and collected in $10-\mathrm{mL}$ polypropylene tubes (Sarstedt, Nuembrecht, Germany). Within 2 h the CSF was centrifuged at $1,800 \mathrm{~g}$ for $10 \mathrm{~min}$ at $4{ }^{\circ} \mathrm{C}$. A small amount of CSF was used for routine analysis, including leukocytes and erythrocytes, glucose and total protein. CSF was frozen at $-80^{\circ} \mathrm{C}$ and sent on dry ice to the Clinical Chemistry Laboratory of the VU University Medical Center. At arrival the status of the samples was checked and stored at $-20^{\circ} \mathrm{C}$ or $-80^{\circ} \mathrm{C}$ until further analysis, usually within a month after arrival. After analysis the remaining CSF was stored at $-80^{\circ} \mathrm{C}$ for future research purposes. CSF $\mathrm{A} \beta$, total $\tau$ and $\mathrm{p}-\tau$ were measured with ELISAs (Innotest $\beta$-amyloid (1-42), Innotest TAUAg and Innotest Phosphotau (181P), Innogenetics, Ghent, Belgium) as described previously [18]. The intra-assay coefficient of variation $(\mathrm{CV}$; mean $\pm \mathrm{SD})$ was $2.0 \pm 0.5 \%$ for $\mathrm{A} \beta, 3.2 \pm 1.3 \%$ for $\tau$ and $2.9 \pm$ $0.8 \%$ for $\mathrm{p}-\tau$ as calculated from averaging coefficients of variation of duplicates of 5 runs randomly selected over 2 years. The inter-assay $\mathrm{CV}($ mean \pm SD) was $10.9 \pm 1.8 \%$ for $\mathrm{A} \beta, 9.9 \pm 2.1 \%$ for $\tau$ and $9.1 \pm$ $1.8 \%$ for $\mathrm{p}-\tau$. Cutoff levels for normal biomarker values were for $\mathrm{A} \beta$ $>550 \mathrm{pg} / \mathrm{mL}$, for total $\tau<375 \mathrm{pg} / \mathrm{mL}$ and for $\mathrm{p}-\tau<52 \mathrm{pg} / \mathrm{mL}[2]$.

\section{Outcome Measures}

Physicians were given the CSF levels and a CSF diagnosis. When all 3 biomarkers had levels outside the normal ranges, the CSF profile was labeled "fits AD." In case only total $\tau$ and $p-\tau$ levels were outside the normal ranges, the CSF profile was labeled "probably fits AD." When $A \beta$ levels were outside the normal range and normal values for the $\tau$ parameters were found, this was labeled "probably does not fit AD." Normal values for all biomarkers was termed "does not fit AD." Combining this CSF diagnosis and the preCSF diagnosis resulted in a postCSF diagnosis.

Physicians were asked to judge the CSF diagnosis as either helpful or not. Helpful meant either increasing their diagnostic certainty or questioning the diagnosis resulting in a change of medical policy. Not helpful meant no important change in diagnostic certainty or change in medical policy.

The total $\tau / A \beta$ ratio is a simplified CSF diagnosis criterion excluding the $p-\tau$ values. When this ratio exceeds 0.52 , the test results are deemed positive for $\mathrm{AD}$. This cutoff value and test characteristics were described earlier [9]. We calculated this ratio for all patients.

We calculated the sensitivity and specificity of the postCSF diagnosis applying the usual categorizing rules and the total $\tau / A \beta$ ratio. The preCSF diagnosis was the gold standard. 
Table 1. Age, MMSE and MoCA of all subjects and in preCSF diagnosis groups

\begin{tabular}{lccccc}
\hline & All & No AD & MCI & AD & Other etiology \\
\hline Subjects, $n$ & 69 & 14 & 29 & 17 & 9 \\
Age (SD), years & $67.9(8.1)$ & $62.9(8.4)$ & $69.5(5.8)$ & $69.4(7.1)$ & $68.0(12.9)$ \\
MMSE (SD) & $25.2(4.0)$ & $25.8(4.3)$ & $26.5(2.2)$ & $22.1(3.9)$ & $25.7(5.7)$ \\
MoCA (SD) & $22.8(4.8)$ & $26.3(2.6)$ & $23.6(3.7)$ & $21.8(3.8)$ & $18.4(7.7)$ \\
\hline
\end{tabular}

MMSE, Mini Mental State Examination; MoCA, Montreal Cognitive Assessment; CSF, cerebrospinal fluid; AD, Alzheimer's disease; MCI, mild cognitive impairment; SD, standard deviation.

Table 2. Reasons to measure cerebrospinal fluid levels and helpfulness of the results in the three age groups

\begin{tabular}{lrrrl}
\hline & $<66$ & $66-70$ & $>70$ & Total \\
\hline To increase certainty & 6 & 6 & 16 & 28 \\
Differentiation AD/no AD & 29 & 29 & 20 & 78 \\
Helpful & & & & \\
$\quad$ Yes & 28 & 24 & 27 & 79 \\
$\quad$ No & 7 & 11 & 9 & 27 \\
Percent helpful & 80 & 69 & 75 & 75 \\
\hline
\end{tabular}

Statistical Analysis

For statistical analysis the SPSS version 20 statistical package for Windows was used. Results are expressed as numbers, percentages, means or medians, and standard deviations. CSF levels were compared in 2 or more groups using the Wilcoxon or KruskallWallis statistic.

\section{Results}

Between 2010 and 2016, 106 patients underwent an LP. Annually on average 250 patients with cognitive problems or symptoms present themselves in the outpatient memory clinic of our Geriatric Medicine Department. The average percentage of patients undergoing this procedure was around $6.8 \%$ per year.

Baseline diagnoses prior to CSF measures were: NCD 8, amnestic MCI 23 and multidomain 6, $\mathrm{AD} 17, \mathrm{VaD} 1$, Lewy body dementia 2 , frontotemporal dementia 5 , other dementias 1, neurological disorder 2, psychiatric disorder 5 , developmental disorder 1, no diagnosis yet 35 .

Table 1 shows age, Mini Mental State Examination and Montreal Cognitive Assessment of the NCD, MCI, AD groups, and the group having another dementia etiology. The mean age for all patients was 67.9 years. No significant age differences between groups were found. Thirty-
Table 3. Number of patients in pre- and postCSF diagnosis groups

\begin{tabular}{lcc}
\hline & preCSF & postCSF \\
\hline No AD & 14 & 16 \\
MCI & 29 & 12 \\
AD & 17 & 56 \\
Other etiology & 9 & 8 \\
\hline Total & 69 & 92 \\
\hline
\end{tabular}

CSF, cerebrospinal fluid; AD, Alzheimer's disease; $\mathrm{MCI}$, mild cognitive impairment.

five patients were younger than 66 years, 35 were in between 66 and 70, and 36 were over the age of 70 .

Table 2 shows in 3 age categories the reasons to measure CSF biomarkers and the number of patients with CSF results that were helpful to the physician.

In the $<66$ and the 66-70 group in 29 out of 35 patients CSF biomarkers were used to differentiate between AD and no $\mathrm{AD}$. In the $>70$ group in 16 out of 36 patients there was already a diagnosis of $\mathrm{AD}$ but more certainty was required by the physician and/or the patient. For all age groups in 78 out of 106 patients CSF biomarkers were used to differentiate between $\mathrm{AD}$ and no AD. The CSF diagnosis was for all age groups helpful to the physicians in $75 \%$ of cases.

Using the CSF tool the following postCSF diagnoses were made, in parentheses the preCSF numbers are given: NCD 8 (8), amnestic 7(23) and multidomain MCI 5 (6), $\mathrm{AD} 56$ (17), $\mathrm{AD}+\mathrm{VaD} 1$ (0), Lewy body dementia 3 (2), frontotemporal dementia 3 (1), neurological disorder 4 (2), psychiatry 7 (5), developmental disorder 1 (1), no diagnosis yet 10 (35). These diagnoses are summarized in 4 postCSF groups in Table 3.

In $61 \%$ of cases the preCSF diagnosis was changed into another diagnosis after CSF. In the majority of these cases this is related to the MCI diagnosis being changed into 
Table 4. Levels of $A \beta$, total $\tau$ and $p-\tau(\mathrm{pg} / \mathrm{mL})$ expressed as medians (IQR) in 2 preCSF diagnosis groups and in 3 age categories

\begin{tabular}{llll}
\hline & A $\beta$ & Total $\tau$ & p- $\tau$ \\
\hline PreCSF diagnosis no AD & & & \\
All ages & $825(572-1,133)$ & $290(157-609)$ & $49(30-71)$ \\
Age $<66(7)$ & $969(808-1,109)$ & $163(140-216)$ & $30(28-49)$ \\
Age 66-70 (5) & $605(450-1,026)$ & $604(390-908)$ & $68(62-122)$ \\
Age $>70(2)$ & 954 & 699 & 97 \\
Study 1 (all ages; $n=18)$ & $759(654-1,030)$ & $367(218-434)$ & $52(36-63)$ \\
\hline PreCSF diagnosis AD & & & \\
All ages & $516(378-625)$ & $659(384-1,057)$ & $79(58-127)$ \\
Age $<66(4)$ & $474(318-617)$ & $661(328-1,403)$ & $98(57-142)$ \\
Age 66-70 (7) & $516(411-550)$ & $621(411-1,159)$ & $81(59-108)$ \\
Age $>70(6)$ & $541(403-651)$ & $691(376-1,083)$ & $76(53-140)$ \\
Study 1 (all ages; $n=47)$ & $474(335-602)$ & $482(393-853)$ & $77(50-103)$ \\
\hline
\end{tabular}

Cutoff levels: $\mathrm{A} \beta>550 \mathrm{pg} / \mathrm{mL}$, total $\tau<375 \mathrm{pg} / \mathrm{mL}, \mathrm{p}-\tau<52 \mathrm{pg} / \mathrm{mL}$. $\mathrm{A} \beta$, total $\tau$ and $\mathrm{p}-\tau$ levels in study 1 , expressed as medians (interquartile range, IQR) have identical cutoff levels. Cerebrospinal fluid (CSF) levels for separate age groups were not available in this study.

the diagnosis $\mathrm{AD}$, and to 25 out of 35 cases without a diagnosis before CSF receiving a postCSF diagnosis.

In Table 4 the CSF levels for the no-AD and $\mathrm{AD}$ groups are presented. Additionally, the results of our previous study are given [5].

\section{A $\beta$ Levels}

In the no- $\mathrm{AD}$ group the median $\mathrm{A} \beta$ levels for each age group were in the normal range with the highest levels in the youngest age group. In the $\mathrm{AD}$ group median $\mathrm{A} \beta$ levels in all age groups were in the pathological range, showing increasing median levels with increasing age. All differences were statistically not significant, Kruskal-Wallis test for the no- $\mathrm{AD}$ and $\mathrm{AD}$ group, $p>0.3$.

\section{Total $\tau$ Levels}

The median total $\tau$ level in the no- $\mathrm{AD} /$ youngest age group was in the normal range as expected, in the other age groups median levels were in the pathological range. In the $\mathrm{AD}$ group all levels were in the pathological range, Kruskal-Wallis statistic for both diagnosis groups, $p>0.8$.

\section{$p-\tau$ Levels}

The median $\mathrm{p}-\tau$ level in the no- $\mathrm{AD} /$ youngest age group was in the normal range but was out of range for both other age groups. In the $\mathrm{AD}$ group for all age groups $\mathrm{p}-\tau$ levels were in the pathological range with the highest median level in the youngest age group, Kruskal-Wallis test for both diagnosis groups, $p>0.8$.

Diagnostic Impact of CSF Biomarkers in Daily Practice
Comparing median levels between the $<66$ and $66-70$ groups showed in the no-AD group for the total $\tau$ and $\mathrm{p}-\tau$ parameters a significant difference, Wilcoxon statistic, $p=0.003$. Probably this also goes for the $>70$ group in comparison to the youngest age group but due to the small number of $>70$ patients we could not show this statistically. No other significant differences were found.

For the usual categorizing rules to interpret CSF levels using the "fits $\mathrm{AD}$ " category, the sensitivity amounted to $71 \%$, including the "probably fits $\mathrm{AD}$ " category the sensitivity rose to $82 \%$. Specificity for the "no-AD" category was $57 \%$, including the "probably no-AD" category resulted in a specificity of $64 \%$. For the ratio rule sensitivity amounted to $88 \%$ and specificity to $64 \%$.

\section{Discussion}

We studied the added value of CSF biomarkers in a group of patients with mean age under 71 years, in an extensive diagnostic protocol of an outpatient memory clinic of a geriatric medicine department in a communitybased hospital. In 78/106 patients CSF levels were used to decide between the diagnosis $\mathrm{AD}$ and no AD. Especially in the 2 youngest age groups CSF levels were used to differentiate between AD and no AD. Moreover, in 75\% of all cases CSF levels were considered to be helpful to the clinician in making a diagnosis. Comparisons between preCSF and postCSF diagnoses showed that the number 
of $\mathrm{AD}$ diagnoses strongly increased. The number of MCI diagnoses were more than halved which means that CSF helped the clinician to decide that $\mathrm{AD}$ was the underlying disease in this syndrome. Moreover, in 25 out of 35 patients without a clear-cut diagnosis after the initial analysis, a diagnosis could be made using the CSF tool.

In the no-AD group we found a differential age effect in the profile of the total $\tau$ and $p-\tau$ levels where only the youngest age group had normal total $\tau$ and $p-\tau$ levels. In the $\mathrm{AD}$ group the profile in the youngest age group was also more typical on inspection. Due to the small number of patients and large variances in CSF levels it will be a priori difficult to find statistically significant differences. Given these limitations these results suggest that the youngest age group has the most typical CSF profile in both the no- $\mathrm{AD}$ and $\mathrm{AD}$ groups.

Sensitivity and specificity of the total $\tau / A \beta$ ratio were at least as good as the sensitivity and specificity of the usual categorization rule to give a CSF diagnosis, given the limitation of moderate to low percentages probably due to the small number of patients.

This is our second study regarding the usefulness of CSF biomarkers in the diagnostic process in an outpatient clinic outside the setting of a research environment. As far as we know no other studies regarding this issue in this particular setting have been published.

In the present study we have attempted to evaluate our policy on the use of CSF biomarkers. We studied clinical usefulness not by measuring change of confidence of the clinician but in terms of helpfulness. This relates in our feeling even more to how a clinician perceives and interprets CSF results. Being less confident may also be helpful in the context of the present study. CSF levels may question the preCSF diagnosis postponing a definite diagnosis preventing unnecessary burdening of patients with an incorrect diagnosis. Being less confident may also urge the physician to find a definite diagnosis by performing extra diagnostic tests. In our first study in $32 \%$ of cases clinicians had gained confidence in the postCSF diagnosis, in $10 \%$ of cases they had less confidence. In the present study the CSF tool was helpful in $75 \%$ of cases. This may also be caused by the high number of preCSF diagnoses in the category "no diagnosis yet," i.e. 35. In 19 of these cases no diagnosis was made. The mean age in these 19 patients was 69 years which may illustrate the difficulty of diagnosing cognitive disorders in elderly subjects burdened by comorbidity and a large overlap in clinical symptoms between normal and pathological aging, thereby making the interpretation of test battery results [19] and MRI [10] more difficult. In the remaining 16 cases with a mean age of 64 years a diagnosis was suspended. This may have been due to the efforts not to diagnose $\mathrm{AD}$ without enough confirmation.

In study 1 the percentage of change between pre- and postCSF diagnosis was $10 \%$. The $61 \%$ in the present study is much higher due to the fact that an LP was performed because of diagnostic doubts. In the first study an LP was performed in consecutive patients regardless of the measure of diagnostic doubt in the preCSF diagnosis.

Given the fact that the number of patients is small and variability of CSF levels between patients is large, we feel nonetheless that our results suggest in comparison to our first study a clearer cutoff level of the patients' age to use CSF as a diagnostic tool, i.e. 65 years. In our youngest age group in both the $\mathrm{AD}$ and no-AD groups CSF levels were typical for the respective diagnoses. This was not the case for both groups $>66$ years. The only statistically significant differences in the present study were found for the total $\tau$ and $p-\tau$ parameters in the no-AD group, comparing the $<66$ and $66-70$ age groups.

Unfortunately, we could not compare the results of both our studies due to changes in the technique of measuring CSF levels between 2006 and 2016. However, we found comparable test results in both studies. This underlines the reliability of the LP procedure and the local processing of the CSF material before transport to the VU University medical center Clinical Chemistry Laboratory.

The weakness of this study is the small number of patients. This is due to the strict inclusion criteria and the nonacademic setting of the study. Patients and physicians are less likely to choose the use of the CSF tool to attain maximal diagnostic certainty despite the fact that the procedure was well tolerated, in agreement with the study of Duits et al. [20].

We conclude by corroborating our earlier results that in our outpatient memory clinic setting CSF biomarkers are a useful diagnostic tool in the diagnostic program. We also confirm the suggestion of the first study that the CSF biomarker tool can best be used in younger subjects. Based on our results a cutoff of 65 years seems a sound criterium for applying this instrument.

Given the usefulness of the CSF tool it would be worthwhile to study this in a much larger study population outside the typical research environment. This would become even more relevant when CSF biomarkers could reliably be measured in plasma instead of in cerebral spinal fluid [21-24].

Published in Celebration of the 30th Anniversary of the inception of Dementia and Geriatric Cognitive Disorders 1990-2020. 


\section{Statement of Ethics}

The study protocol has been approved by the local ethics' committee. All patients gave their written informed consent.

\section{Disclosure Statement}

The authors declare no conflicts of interest for this article.

\section{Funding Sources}

No funding has been received.

\section{Author Contributions}

L. Boelaarts designed this study, performed the lumbar punctures, built the database and collected and analyzed the data, and wrote this article. J.F.M. de Jonghe assisted in the design of the study, in analyzing the data and writing this article. P. Scheltens assisted in the design of the study, interpreting the CSF biomarker levels and in writing the article.

\section{References}

1 Blennow K, Hampel H. CSF markers for incipient Alzheimer's disease. Lancet Neurol. 2003 Oct;2(10):605-13.

2 Mulder C, Verwey NA, van der Flier WM, Bouwman FH, Kok A, van Elk EJ, et al. Amyloid- $\beta$ (1-42), total tau, and phosphorylated tau as cerebrospinal fluid biomarkers for the diagnosis of Alzheimer disease. Clin Chem. 2010 Feb;56(2):248-53.

3 Dubois B, Feldman HH, Jacova C, Hampel H, Molinuevo JL, Blennow K, et al. Advancing research diagnostic criteria for Alzheimer's disease: the IWG-2 criteria. Lancet Neurol. 2014 Jun;13(6):614-29.

4 Jack CR Jr, Bennett DA, Blennow K, Carrillo MC, Dunn B, Haeberlein SB, et al.; Contributors. NIA-AA Research Framework: toward a biological definition of Alzheimer's disease. Alzheimers Dement. 2018 Apr;14(4):535-62.

5 Kester MI, Boelaarts L, Bouwman FH, Vogels RL, Groot ER, van Elk EJ, et al. Diagnostic impact of CSF biomarkers in a local hospital memory clinic. Dement Geriatr Cogn Disord. 2010;29(6):491-7.

6 Slats D, Spies PE, Sjögren MJ, Visser PJ, Verbeek MM, Rikkert MG, et al. CSF biomarker utilisation and ethical considerations of biomarker assisted diagnosis and research in dementia: perspectives from within the European Alzheimer's Disease Consortium (EADC). J Neurol Neurosurg Psychiatry. 2010 Jan;81(1):124-5.

7 Spies PE, Slats D, Ramakers I, Verhey FR, Olde Rikkert MG. Experiences with cerebrospinal fluid analysis in Dutch memory clinics. Eur J Neurol. 2011 Jul;18(7):1014-6.

8 Skillbäck T, Farahmand BY, Rosén C, Mattsson N, Nägga K, Kilander L, et al. Cerebrospinal fluid tau and amyloid- $\beta 1-42$ in patients with dementia. Brain. 2015 Sep;138(Pt 9): 2716-31.

9 Duits FH, Teunissen CE, Bouwman FH, Visser PJ, Mattsson N, Zetterberg H, et al. The cerebrospinal fluid "Alzheimer profile": easily said, but what does it mean? Alzheimers Dement. 2014 Nov;10(6):713-723.e2.
10 Boelaarts L, Scheltens P, de Jonghe J. Does MRI increase the diagnostic confidence of physicians in an outpatient memory clinic? Dement Geriatr Cogn Disord Extra. 2016 Jun; 6(2):242-51.

11 McKhann GM, Knopman DS, Chertkow H, Hyman BT, Jack CR Jr, Kawas CH, et al. The diagnosis of dementia due to Alzheimer's disease: recommendations from the National Institute on Aging-Alzheimer's Association workgroups on diagnostic guidelines for Alzheimer's disease. Alzheimers Dement. 2011 May;7(3):263-9.

12 Neary D, Snowden JS, Gustafson L, Passant U, Stuss D, Black S, et al. Frontotemporal lobar degeneration: a consensus on clinical diagnostic criteria. Neurology. 1998 Dec;51(6): 1546-54.

13 Román GC, Tatemichi TK, Erkinjuntti T, Cummings JL, Masdeu JC, Garcia JH, et al. Vascular dementia: diagnostic criteria for research studies. Report of the NINDS-AIREN International Workshop. Neurology. 1993 Feb;43(2):250-60.

14 McKeith IG, Dickson DW, Lowe J, Emre M, O'Brien JT, Feldman H, et al.; Consortium on DLB. Diagnosis and management of dementia with Lewy bodies: third report of the DLB Consortium. Neurology. 2005 Dec;65(12): 1863-72.

15 Petersen RC, Smith GE, Waring SC, Ivnik RJ, Tangalos EG, Kokmen E. Mild cognitive impairment: clinical characterization and outcome. Arch Neurol. 1999 Mar;56(3):303-8.

16 Petersen RC, Stevens JC, Ganguli M, Tangalos EG, Cummings JL, DeKosky ST. Practice parameter: early detection of dementia: mild cognitive impairment (an evidence-based review). Report of the Quality Standards Subcommittee of the American Academy of Neurology. Neurology. 2001 May;56(9):1133-42.

17 Fazekas F, Chawluk JB, Alavi A, Hurtig HI, Zimmerman RA. MR signal abnormalities at $1.5 \mathrm{~T}$ in Alzheimer's dementia and normal aging. AJR Am J Roentgenol. 1987 Aug;149(2): 351-6.
18 Jongbloed W, Kester MI, van der Flier WM, Veerhuis R, Scheltens P, Blankenstein MA, et al. Discriminatory and predictive capabilities of enzyme-linked immunosorbent assay and multiplex platforms in a longitudinal Alzheimer's disease study. Alzheimers Dement. 2013 May;9(3):276-83.

19 Boelaarts L, Meyer SR, Scheltens P, de Jonghe JF. Testing episodic memory in elderly subjects:; not as simple as it looks. Dement Geriatr Cogn Disord Extra. 2019 Jun;9(2):207-16.

20 Duits FH, Martinez-Lage P, Paquet C, Engelborghs S, Lleó A, Hausner L, et al. Performance and complications of lumbar puncture in memory clinics: results of the multicenter lumbar puncture feasibility study. Alzheimers Dement. 2016 Feb;12(2):154-63.

21 Blennow K, De Meyer G, Hansson O, Minthon L, Wallin A, Zetterberg $\mathrm{H}$, et al.; KNDStudy Group. Evolution of Abeta42 and Abeta 40 levels and Abeta42/Abeta 40 ratio in plasma during progression of Alzheimer's disease: a multicenter assessment. J Nutr Health Aging. 2009 Mar;13(3):205-8.

22 Hansson $\mathrm{O}$, Zetterberg $\mathrm{H}$, Vanmechelen $\mathrm{E}$ Vanderstichele H, Andreasson U, Londos E, et al. Evaluation of plasma Abeta(40) and Abeta(42) as predictors of conversion to Alzheimer's disease in patients with mild cognitive impairment. Neurobiol Aging. 2010 Mar; 31(3):357-67.

23 Jongbloed W, Herrebout MA, Blankenstein MA, Veerhuis R. Quantification of clusterin in paired cerebrospinal fluid and plasma samples. Ann Clin Biochem. 2014 Sep;51(Pt 5): 557-67.

24 Duits FH, Hernandez-Guillamon M, Montaner J, Goos JD, Montañola A, Wattjes MP, et al. Matrix metalloproteinases in Alzheimer's disease and effects of concurrent cerebral microbleeds. J Alzheimers Dis. 2015;48(3): $711-20$. 